\title{
Laterally Actuated Torsional Micromirrors for Large Static Deflection
}

\author{
Veljko Milanović, Member, IEEE, Matthew Last, and Kristofer S. J. Pister
}

\begin{abstract}
We report on the implementation of laterally electrostatically actuated, torsionally suspended silicon-on-insulator (SOI) micromirrors with a static optical deflection angle of over $40^{\circ}$ peak-to-peak. Decoupling the actuator and mirror design allows for large actuator arrays, allowing large de deflection angle and high resonant frequency to coexist in the same device. The micromirror structures are fully monolithic, micromachined from the front side and back side of an SOI wafer-device layer. In-plane actuation is transformed into out-of-plane motion and rotation, enabling integration of a wide variety of SOI-MEMS sensors, actuators, and micromirrors. When operated in resonance at $1321 \mathrm{~Hz}$, a typical device measured up to $92^{\circ}$ peak-to-peak optical deflection at 127 Vdc with 15 Vac amplitude.
\end{abstract}

Index Terms-Comb drive, high aspect ratio, MEMS, microfabrication, micromachining, micromirror, MOEMS, optical MEMS, static deflection.

\section{INTRODUCTION}

$\mathbf{T}$ HE RECENT focus of the MEMS world on optical applications of micromachined devices has pushed the field out of surface micromachining technology [1]-[3]. This is mainly due to the need for very flat mirrors and the desire for the large actuation forces available using high aspect-ratio micromachining. By moving to silicon-on-insulator (SOI) technology, the flatness issue is mostly ameliorated [4]. The biggest remaining obstacle in SOI MEMS is the demonstration of out-of-plane motion.

This work shows that it is possible to integrate planar high aspect-ratio lateral actuators and position sensors with micromirrors. Mirror design is thereby decoupled from actuator/sensor design. The system designer now has the freedom to choose an appropriate actuator for the desired range of motion, power consumption, and speed. As an additional advantage, the space above and beneath the micromirror is free, not limiting mirror movement as with most vertical electrostatic actuation devices [5]. Although the use of erected/hinged supports for micromirrors were demonstrated to significantly increase angle of rotation [3], [6], the designer is then forced to trade off increased rotation angle with large actuation voltages.

The concept of the lateral actuation is depicted in Fig. 1. By applying force $F$ on the actuating arm (Fig. 1) along the $x$-axis by either pushing or pulling on the arm, the load is transferred along linkages and applied above or below the shear centers

Manuscript received February 15, 2002; revised October 8, 2002.

V. Milanović is with the Adriatic Research Institute, Berkeley, CA 94704-1029 USA.

M. Last and K. S. J. Pister are with the Berkeley Sensor and Actuator Center, University of California, Berkeley, CA 94720 USA.

Digital Object Identifier 10.1109/LPT.2002.806085

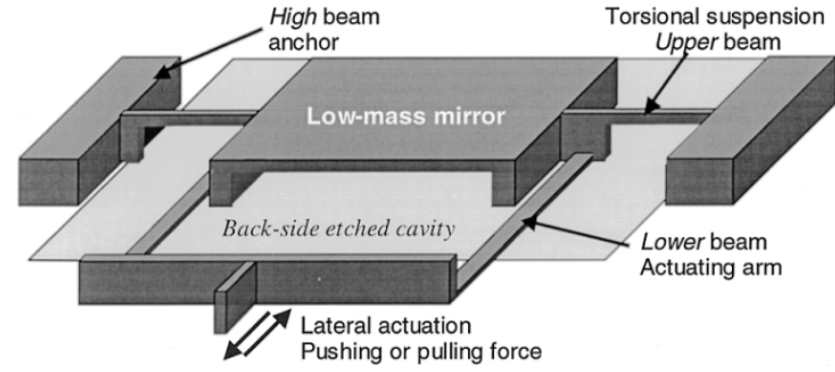

Fig. 1. Conversion of lateral actuation to rotation by applying actuation at points displaced from torsional suspensions' shear centers. Torsional suspensions are etched to Upper-beam level, and actuation arms are etched to Lower-beam level to apply push-pull force transferred from adjacent in-plane actuators (not shown in the figure).
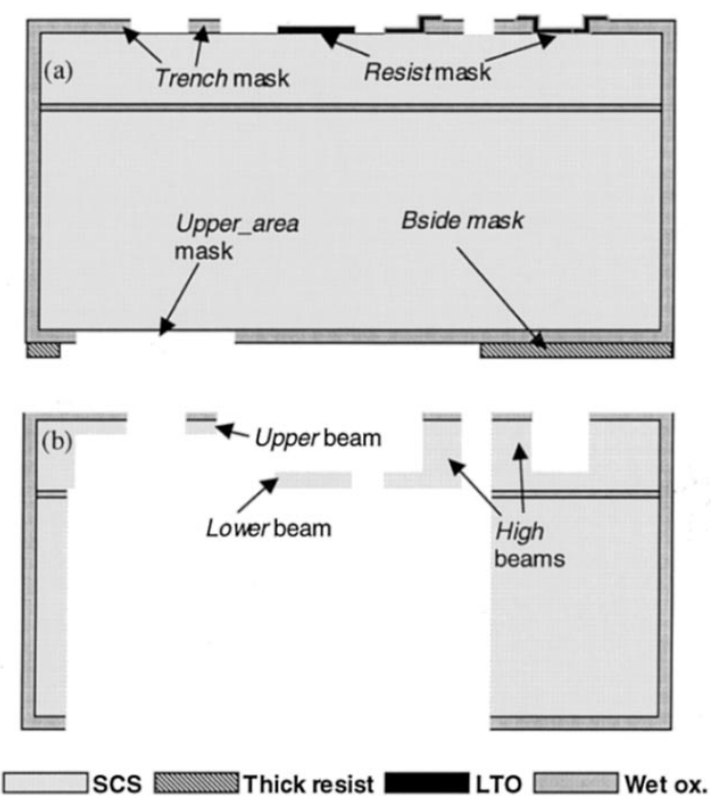

Fig. 2. Fabrication schematic for three-level beam structures. Four masks, two on front- and two on back-side are prepared first, followed by back- and front-side DRIE high aspect ratio silicon etches.

(axes of rotation) of the mirror torsion beams, resulting in rotation. The key innovation that allows lateral actuation to be transformed into out-of-plane rotation is the micromachining of beams out of a monolithic slab of Silicon whose shear centers are displaced vertically from the center of the SOI device layer. For better understanding of the designs and the fabrication process, we have labeled these beams Upper, Lower, and High, as seen in Figs. 1 and 2.

Realization of the proposed concept requires selective, high aspect ratio multilevel etching [7] of single crystal Silicon (SCS) 
in SOI wafers, using deep reactive ion etching (DRIE) [8]. The timed multilevel etch results in various types of beams providing the needed distance between shear axes and loads.

\section{DESIGN CONSIDERATIONS}

The four mask process described in Section III provides the designer with substantial flexibility. The scanning mirror design problem can be broken down into three coupled design problems: the lateral to rotational mechanism, the actuator(s), and the mirror itself.

The performance of the whole system follows from the design of the mirror. Here, the issues are radius of curvature, dynamic deflection, and moment of inertia. While the bare SCS will be quite flat, the addition of a front-surface metallization to enhance reflectivity degrades flatness due to residual stress in the metal and thermal expansion mismatch between the metal and the silicon. Radius of curvature can be improved by increasing the mirror thickness.

Angular acceleration of the mirror is limited by the moment of inertia of the mirror. The moment of inertia can be minimized without sacrificing mirror rigidity by using tall and thin reinforcing ribs behind a thin mirror surface. Once the mirror moment of inertia is known, the displacement bandwidth then sets a lower limit on the torsional stiffness of the support structure. Together with the desired scan angle, these form the primary constraints on the lateral-to-rotational coupling between the actuators and the mirror.

The torsional stiffness of the suspension is a combination of the primary torsional supports of the mirror, and the torsional stiffness of the linkage Lower (see Fig. 1). This stiffness is nonlinear, increasing dramatically in tension, and decreasing in compression. This effect can be minimized by proper choice of the attachment point of the linkage along the length of the mirror. This attachment point need not be directly above the attachment point of the torsion beam, and in particular can be further away from the actuator input. In this case, a geometric nonlinearity in the relationship between input force and resulting moment counteracts the effect of the nonlinear spring-stiffening in the beam.

Finally, the actuator must supply enough force to rotate the mirror in the presence of the above nonlinearities, as well as enough displacement to handle both the rotation of the attachment point and the lateral deflection of the torsion beams. Both of these displacements are also nonlinear functions of the input force. For the large mirrors, large angles, and fast response times required of many current micromirror designs, relatively large comb drives are required. The electrostatic stability of the comb drives places a constraint on the comb suspension.

\section{MultileVel BeAm SOI-MEMS FABRicATION}

Implementing the concept described above requires the capability of fabricating Si beams at different vertical levels in an SOI wafer. The basic methodology for creating multilevel beam SOI MEMS is depicted in Fig. 2, and is described in more detail elsewhere [9]. Namely, combination of front-side and back-side timed multilevel etches [7] of the same slab of single crystal Si results in Si beams at different vertical levels. Two masks from the front side allow for two depths of etching (Trench and Resist mask in Fig. 2) - these deep and shallow trenches define full thickness beams, labeled "High," and thinned-down beams, labeled "Lower," respectively, in Fig. 2. Thin beams can be also created close to the surface of the SOI device layer by etching from the back side of the wafer; these are labeled "Upper" in the figure. The micromirrors demonstrated to date and reported in this work utilize all three beams, i.e., Upper, Lower, and High, and only require three masks for beam fabrication and one mask for removing handle wafer $\mathrm{Si}$ from back side of the shown device layer for a total of four masks, as shown in Fig. 2.

Fabrication is done on SOI wafers: double-side polished, around $50-\mu \mathrm{m}$ device layer SCS, $1-\mu \mathrm{m}$ insulating thermal oxide, and 300- $\mu \mathrm{m}$ handle wafer SCS. The wafer first undergoes a 1.4- $\mu \mathrm{m}$ wet thermal oxidation (wet ox in Fig. 2). The Trench mask for deep front-side trenches is then etched into the oxide on the front side of the wafer, stopping on silicon. Then, $600 \mathrm{~nm}$ of low-pressure chemical vapor deposition low-temperature oxide [(LTO) in Fig. 2] is deposited on the wafer and annealed. The second mask (defining Lower) is etched into the LTO, again stopping on silicon. The front side of the wafer is now ready for DRIE.

On the back side of the wafer, two masks are employed. The first mask defines Upper_area, i.e., areas where device SCS will be thinned from below achieving a thin Upper beam. This masks the back-side etch through the thermal oxide. Then, a second back-side mask, Bside, is applied using thick resist. Etching of the back side of the wafer is performed first. The first back-side etch is timed, to about 70- $\mu \mathrm{m}$ depth. Oxide RIE removes the Upper_area oxide mask. Silicon DRIE resumes, terminating when the deeper trenches reach the insulating oxide. At this point, the areas exposed by Upper_area mask have reached the oxide while the rest of the back-side etch area has about $60 \mu \mathrm{m}$ of handle-wafer silicon remaining. The insulating oxide is thinned from $1 \mu \mathrm{m}$ to $7000 \mathrm{~A}$ in those areas. Then the remaining back-side DRIE is done until all back-side trenches reach the oxide. Effectively, the two masks, Upper_area and Bside, have been transferred onto the insulating oxide that now has two thicknesses, approximately $1 \mu \mathrm{m}$ and 3000-5000 A. This difference is used to etch into the back of the devices, thinning the device SCS from below. Using this etch, thin torsion beams and low-mass mirrors can be made.

On the front side, first a timed DRIE is done through approximately half the device layer thickness, $\sim 25 \mu \mathrm{m}$. At this point, oxide RIE on the front side removes the Shallow-trench protection oxide $(600 \mathrm{~nm})$ also thinning the wet thermal oxide. This etch is timed to completely remove the thinner oxide layer while preserving at least 4000-5000 A of thermal oxide for masking the second DRIE. The second DRIE is done until the devices are complete, i.e., until the bottom of device layer is reached on every structure, especially small-featured structures such as comb-drive fingers, etc., and until the Lower beams are thinned to desired height, e.g., $8-12 \mu \mathrm{m}$. Since our designs position back-side trenches under all moving structures, the wafer, at this point, has fully released and functional MEMS ready for testing. The final step is to remove the oxide masks using an HF acid dip, leaving a smooth and flat $\mathrm{Si}$ mirror surface as shown in Fig. 3. 


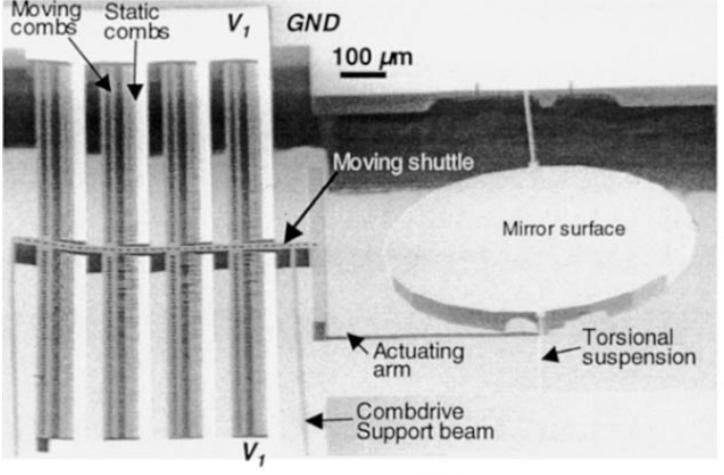

(a)

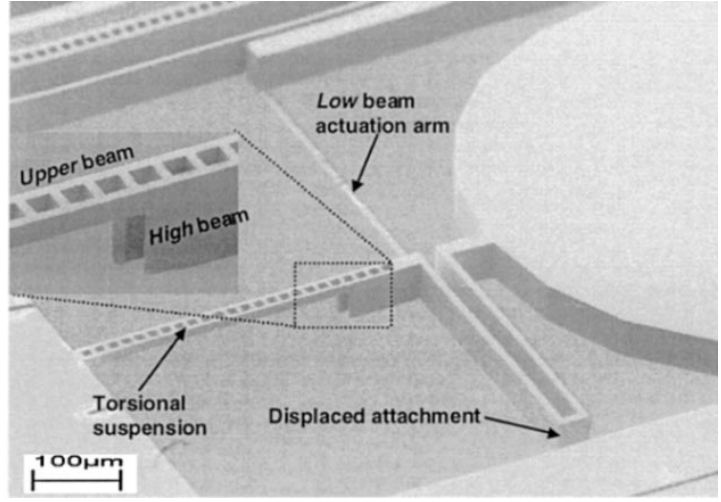

(b)

Fig. 3. Scanning electron microscopes of fabricated devices. (a) One degree-of-freedom mirror designed for push mode operation which measured up to $11^{\circ}$ mechanical rotation. (b) Three beam thicknesses available in this process, as well as the novel perforated torsional suspension beam which maximizes torsional compliance while maintaining lateral stiffness. This device utilizes the type of comb drive shown in (a), but actuating in the opposite direction (away from the mirror, i.e., pulling).

\section{CHARACTERIZATION AND DISCUSSION}

The devices characterized in this work were designed and fabricated to be actuated by electrostatic comb drives. The comb drives consisted of 560 comb fingers with $4 \mu \mathrm{m}$ gaps made from High beams configured to push or pull on the actuating arms connecting them to the mirror. For devices with bidirectional rotation, $280 \mathrm{comb}$ fingers were configured to push while 280 fingers would pull.

To test the static deflection as a function of voltage, the wafer was placed on a probe station and individual devices were electrically contacted with probes to which a varying dc bias from 0 to $130 \mathrm{~V}$ was applied. A laser beam was deflected from the mirror surface onto a wall at a known distance from the device. Dc deflection for a pull-mode device was up to $22^{\circ}$ optical, while for a device in push-mode, $21^{\circ}$ was achieved. A laser Doppler vibrometer is used to obtain the frequency response of a single device equipped with both push-type and pull-type actuators. The first resonant mode is greater than $2 \mathrm{kHz}$ and increases with the bias voltage applied to the actuators. This occurs in both push and pull mode and appears to be due to spring stiffening in the mirror suspension.

Interferometric measurements of individual mirrors showed radii of curvature greater than $4 \mathrm{~m}$ and rms roughness below $30 \mathrm{~nm}$.

\section{CONCLUSION}

The combination of back- and front-side multilevel etches allows for a new kind of high aspect ratio MEMS that combines in-plane actuation with out-of-plane motion. One obvious application area is in MEMS micromirrors. The methodology is also being applied for microrobotics applications where out-of-plane actuation is utilized in robot motion. Our current focus is on improving and optimizing the mechanical design of the micromirrors in current technology, incorporating etch-stop methodologies to eliminate timed etches and improve yield, and exploring a new technology by applying bonding and transfer to include additional beam levels.
The demonstrations of the proposed methodology have to date been limited to one-axis micromirror scanning. However, we believe that it extends to two-axis applications by use of gimbal suspensions. While work along that direction has been initiated and preliminary structures fabricated [10], a conclusive demonstration remains our future work.

\section{ACKNOWLEDGMENT}

The authors wish to thank C. Keller and L. Zhou for many useful technical discussions and assistance with device fabrication.

\section{REFERENCES}

[1] M. C. Wu, L. Y. Lin, S. S. Lee, and C. R. King, "Free-space integrated optics realized by surface-micromachining," Int. J. High Speed Electron. Syst., vol. 8, no. 2, pp. 283-297, 1997.

[2] M. Last and K. S. J. Pister, "2-DOF actuated micromirror designed for large DC deflection," presented at the MOEMS'99, Mainz, Germany, Aug. 29-Sept. 1, 1999.

[3] P. R. Patterson, G. J. Su, H. Toshiyoshi, and M. C. Wu, "A MEMS 2-D scanner with bonded single-crystalline honeycomb micromirror, late news," in Proc. Solid-State Sensor and Actuator Workshop, Hilton Head, SC, June 2000, pp. 17-18.

[4] R. Conant, J. Nee, K. Y. Lau, and R. S. Muller, "A flat high-frequency scanning micromirror," in Proc. Solid-State Sensor and Actuator Workshop, Hilton Head, SC, June 2000, pp. 6-9.

[5] J. E. Ford, V. A. Aksyuk, D. J. Bishop, and J. A. Walker, "Wavelength add-drop switching using tilting micromirrors," J. Lightwave Technol., vol. 17, pp. 904-911, May 1999.

[6] H. Toshiyoshi, W. Piyawattanametha, C.-T. Chan, and M. C. Wu, "Linearization of electrostatically actuated surface micromachined 2-D optical scanner," J. Microelectromech. Syst., vol. 10, pp. 205-214, June 2001.

[7] Y. Mita, M. Mita, A. Tixier, J.-P. Gouy, and H. Fujita, "Embeddedmask-methods for mm-scale multi-layer vertical/slanted Si structures," in Proc. IEEE 13th Annu. Int. Conf. Micro Electro Mech. Syst., Miyazaki, Japan, Jan. 23-27, 2000.

[8] R. Bosch Gmbh, "Trench etch process for a single-wafer RIE dry etch reactor," US Patent 4855 017, Sept. 8, 1988.

[9] V. Milanović, "Multilevel beam SOI-MEMS for optical applications," in Proc. 9th IEEE Int. Conf. Electron., Circuits and Syst., Dubrovnik, Croatia, Sept. 2002, pp. 281-285.

[10] V. Milanović, M. Last, and K. S. J. Pister, "Torsional micromirrors with lateral actuators," in Proc. 11th Int. Conf. Solid State Sensors and Actuators-Transducers '01, Munich, Germany, June. 2001, pp. 1298-1301. 